\title{
Apolipoprotein E and adiponectin levels in coronary artery disease patients with low-density lipoprotein cholesterol and non-high-density lipoprotein cholesterol discordance
}

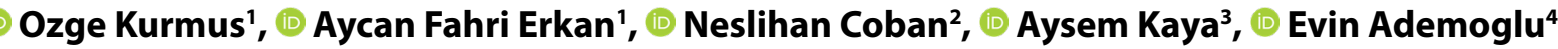 \\ 'Department of Cardiology, Ufuk University, Faculty of Medicine, Ankara, Turkey \\ 2Department of Genetics, Istanbul University, Aziz Sancar Institute for Experimental Medicine, Istanbul, Turkey \\ ${ }^{3}$ Department of Biochemistry Laboratory, Istanbul University-Cerrahpasa, Institute of Cardiology, Istanbul, Turkey \\ ${ }^{4}$ Department of Biochemistry, Istanbul University, Faculty of Medicine, Istanbul, Turkey
}

\begin{abstract}
Objectives: The aim of this study was to investigate whether serum apolipoprotein $E(A p o E)$ and adiponectin were associated with discordance of low-density lipoprotein cholesterol (LDL-C) and non-high-density lipoprotein cholesterol (non-HDL-C) levels in coronary artery disease (CAD) patients.

Methods: A total of 243 patients with significant CAD were studied. The fasting levels of serum lipids, plasma ApoE, and adiponectin were measured. The median LDL-C and non-HDL-C values were determined to assess and analyze discordance. Discordance was defined as an LDL-C $\geq$ the median and non-HDL-C $<$ the median, or an LDL-C $<$ the median and a non-HDL-C $\geq$ the median. ApoE and adiponectin were compared between discordant and concordant groups.

Results: Discordance between the LDL-C and the non-HDL-C was observed in $14 \%$ of the patients. Although the median adiponectin was lowest in the group with an $\mathrm{LDL}-\mathrm{C}<$ median and a non-HDL-C $\geq$ the median, and the median $\mathrm{ApoE}$ was lowest in the group with an LDL-C $\geq$ median and a non-HDL-C $<$ the median, no statistically significant differences in the ApoE and adiponectin were recorded between the groups ( $p=0.186$ and $p=0.161$, respectively). Adiponectin was negatively correlated with triglyceride and remnant cholesterol $(p<0.001$ and $p<0.001$, respectively).

Conclusion: While discordance between the LDL-C and the non-HDL-C in CAD patients (14\%) was observed, the plasma ApoE and adiponectin levels were not significantly different between the discordant and concordant groups.

Keywords: Adiponectin, apolipoprotein E, coronary artery disease
\end{abstract}

A therosclerosis is a multifactorial disorder and lipoprotein abnormalities are important in the pathogenesis of the disease. A subset of patients with either normal lipid levels or low levels of low-density lipoprotein cholesterol (LDL-C) following treatment still experiences atherosclerotic events [1]. Other hidden lipoprotein alterations may have a potential role in the pathogenesis of atherosclerosis.

Apolipoprotein $E(A p o E)$, predominantly synthesized in the liver, is essential for lipid metabolism. It promotes the clearance of remnants of triglyceride-rich lipoproteins TRLs (TRLs) from the circulation into the liver [2]. ApoE is not a constituent of the LDL particle $[3,4]$. ApoE, although generally considered antiatherogenic, is associated with metabolic syndrome and ApoE-rich lipoprotein particles are atherogenic [5-7].

Adiponectin is secreted from adipose tissue and displays antiatherogenic and anti-inflammatory properties $[8,9]$. It is linked to obesity, diabetes mellitus and dyslipidemia [10-12].

LDL-C and non-high-density lipoprotein cholesterol (non$\mathrm{HDL}-\mathrm{C}$ ) are main targets for lipid-lowering therapy to prevent atherosclerotic events. However, not all patients have concor-

Address for correspondence: Ozge Kurmus, MD. Department of Cardiology, Ufuk University, Faculty of Medicine, Ankara, Turkey

Phone: +90 3122044000 E-mail: ozge_kurmus@yahoo.com ORCID: 0000-0003-2067-7492

Submitted Date: January 22, 2021 Accepted Date: February 08, 2021 Available Online Date: March 22, 2021

${ }^{\circ}$ Copyright 2021 by International Journal of Medical Biochemistry - Available online at www.internationalbiochemistry.com

OPEN ACCESS This work is licensed under a Creative Commons Attribution-NonCommercial 4.0 International License. 
dant LDL-C and non-HDL-C levels. Reports have suggested that a sizeable proportion of patients have a low LDL-C and a high non-HDL-C level, or a high LDL-C and a low non-HDL-C level [13-15]. The objective of this study was to investigate whether the serum ApoE and serum adiponectin values were associated with discordance of the LDL-C and non-HDL-C levels in patients with significant coronary artery disease (CAD).

\section{Materials and Methods}

\section{Study design and patients}

This was a cross-sectional study. The research protocol was approved by Istanbul University Faculty of Medicine Clinical Research Ethics Committee on October 13, 2017 (no: 16). Written informed consent was obtained from each patient. Patients referred for coronary angiography with the suspicion of stable CAD were prospectively recruited between June 2018 and January 2019. The coronary angiography procedures were performed through the femoral artery using the standard Judkins method. The baseline diagnostic angiograms of the patients were assessed independently by 2 experienced interventional cardiologists who were blinded to the patients'lipid parameters, and ApoE and adiponectin levels. Epicardial coronary vessel stenosis of $\geq 50 \%$ was considered significant CAD. Diffusion of CAD lesions was assessed by calculating the Gensini score [16]. The exclusion criteria for the study were a history of coronary revascularization, severe valvular disease or heart failure, malignancy, inflammatory disease, active infection, renal or hepatic insufficiency, or hypo/hyperthyroidism. Since statins and peroxisome proliferator-activator receptor agonists have been suggested to effect plasma adiponectin and ApoE levels, patients using a statin, fibrate, or thiazolidinedione were also excluded [17-19]. In all, the study group comprised 243 patients with significant CAD detected during coronary angiography.

The clinical parameters assessed were age, gender, and coronary risk factors. Hypertension was defined as systolic blood pressure $\geq 140 \mathrm{mmHg}$ and/or diastolic blood pressure $\geq 90$ $\mathrm{mmHg}$ and/or current medication with antihypertensive drugs. Patients were classified as diabetic if they had been informed of this diagnosis prior to the study and had been using oral antidiabetic drugs or insulin treatment upon study admission. Body mass index (BMI) was calculated as body weight in kilograms divided by the squared height in meters $\left(\mathrm{kg} / \mathrm{m}^{2}\right)$.

\section{Laboratory measurements}

Lipid measurements were performed using fasting blood samples taken before the angiography. The plasma concentration of total cholesterol, LDL-C, HDL-C, and triglycerides (TG) was measured with a clinical biochemistry analyzer (Architect c8000; Abbott Laboratories, Lake Bluff, IL, USA). The enzymatic colorimetric method was used for quantitative determination of total cholesterol. The endpoint colorimetric method was used for quantitative determination of HDL-C. LDL-C was measured using the quantitative colorimetric method. The glycerol phosphate oxidase method was used to determine a quantitative TG level measurement. The level of non-HDL-C was calculated as total cholesterol minus HDL-C and remnant cholesterol was calculated as non-HDL-C minus LDL-C.

The concentration of ApoE was assessed immunonephelometrically using a BN ProSpec analyzer (Siemens Healthcare Diagnostics Products $\mathrm{GmbH}$, Marburg, Germany). Adiponectin was measured immuneturbidimetrically using a Randox kit (Randox Laboratories Ltd., Crumlin, County Antrim, UK) and a Cobas c501 chemistry analyzer (Roche Diagnostics, Basel, Switzerland).

\section{Statistical analysis}

Categorical variables were defined as percentages. The normality of the distribution of continuous variables was evaluated with the Kolmogorov-Smirnov test or the Shapiro-Wilk test. Continuous data were described as mean \pm SD for normal distributions, and median (interquartile range [IQR]) for skewed distributions. Pearson's or Spearman's correlation analysis was used to examine the correlation between continuous variables of lipid parameters, ApoE, and adiponectin. Differences between the 2 groups were analyzed using the Man$\mathrm{n}$-Whitney $\mathrm{U}$ test. First, the medians for LDL-C and non-HDL-C we determined in order to examine the discordance between them. The patients were categorized into groups of less than or equal to/greater than the median LDL-C and non-HDL-C levels. As there is no standard cutoff point for discordance, the median was selected to define discordance and to make it easier to apply to our study population. Discordance was defined as an LDL-C $\geq$ the median, and non-HDL-C as $<$ the median, or an LDL-C $<$ the median and a non-HDL-C $\geq$ the median. Concordant groups were defined as both the LDL-C and the non-HDL-C $\geq$ the median, or both LDL-C and non-HDL-C $<$ the median. Differences between the baseline patient characteristics across these categories were analyzed with a chi-squared test to compare categorical variables and one-way analysis of variance or the Kruskal-Wallis test for continuous measures. The Bonferroni or Conover-Inman test was performed for binary comparisons between the groups. The data analysis was performed using IBM SPSS Statistics for Windows, Version 22.0 (IBM Corp., Armonk, NY, USA). A p value $<0.05$ was accepted as statistically significant.

\section{Results}

The mean age of the study population was $61.4 \pm 11.6$ years and $53.9 \%$ of the 243 patients were male. The baseline characteristics are presented in Table 1. The mean serum adiponectin concentration was $7.3 \pm 3.9 \mu \mathrm{g} / \mathrm{mL}$ and the mean serum ApoE concentration was $3.9 \pm 2.8 \mathrm{mg} / \mathrm{dL}$.

The LDL-C levels were strongly and positively correlated with the non-HDL-C levels $(r=0.849 ; p<0.001)$, but there was 
Table 1. Baseline characteristics of the study population

\begin{tabular}{ll}
\hline Characteristics & \\
Gender (\% male) & 53.9 \\
Age (years) (mean $\pm \mathrm{SD})$ & $61.4 \pm 11.6$ \\
Smoking (\%) & 41.6 \\
Hypertension (\%) & 45.3 \\
Diabetes $(\%)$ & 36.3 \\
Body mass index $\left(\mathrm{kg} / \mathrm{m}^{2}\right)($ mean $\pm \mathrm{SD})$ & $23.5 \pm 3.8$ \\
Biochemical analysis $($ mean $\pm S D$ or median [interquartile range]) \\
Total cholesterol $(\mathrm{mg} / \mathrm{dL})$ & $198.2 \pm 47.4$ \\
LDL-C (mg/dL) & $117(51)$ \\
HDL-C (mg/dL) & $40.7 \pm 9.5$ \\
Triglycerides $(\mathrm{mg} / \mathrm{dL})$ & $140(95)$ \\
Non-HDL-C $(\mathrm{mg} / \mathrm{dL})$ & $157.4 \pm 45.5$ \\
Fasting & \\
glucose $(\mathrm{mg} / \mathrm{dL})$ & $118.9 \pm 38.7$ \\
Creatinine $(\mathrm{mg} / \mathrm{dL})$ & $0.92 \pm 0.38$ \\
Mean Gensini score & $35.6 \pm 11.1$ \\
Mean adiponectin $(\mu \mathrm{g} / \mathrm{mL})$ & $7.3 \pm 3.9$ \\
Mean ApoE $(\mathrm{mg} / \mathrm{dL})$ & $3.9 \pm 2.8$ \\
\hline
\end{tabular}

ApoE: Apolipoprotein E; HDL-C: High-density lipoprotein cholesterol; LDL-C: Low-density lipoprotein cholesterol.

discordance between them. Discordance of LDL-C and non$\mathrm{HDL}-\mathrm{C}$ values was found in $14 \%$ of the patients. The magnitude of the discordance and the distribution of LDL-C and non-HDL-C levels according to median values are shown in Figure 1. Non-HDL-C was positively correlated with TG $(r=0.474 ; p<0.001)$.

Adiponectin was negatively correlated with both TG and remnant cholesterol $(r=-0.299 ; p<0.001$ and $r=-0.259 ; p<0.001$, respectively). It was positively correlated with $\mathrm{HDL}-\mathrm{C}(\mathrm{r}=0.227$; $\mathrm{p}<0.001)$. Adiponectin was not correlated with LDL-C or nonHDL-C ( $p=0.081$ and $p=0.815$, respectively). ApoE was not correlated any of the lipid parameters. Furthermore, adiponectin was not correlated with ApoE $(p=0.838)$. When the patients were divided into 2 groups according to HDL-C level, the patients with an $\mathrm{HDL}-\mathrm{C} \geq 40 \mathrm{mg} / \mathrm{dL}$ had higher adiponectin levels than patients with an HDL-C $<40 \mathrm{mg} / \mathrm{dL}$ (6.8 [IQR: 5.5$] \mu \mathrm{g} /$ $\mathrm{mL}$ and 4.8 [IQR: 4.7$] \mu \mathrm{g} / \mathrm{mL}$, respectively; $p<0.001$ ). Patients with a TG level $\geq 200 \mathrm{mg} / \mathrm{dL}$ had lower adiponectin levels than patients with a TG $<200 \mathrm{mg} / \mathrm{dL}$ (3.9 [IQR: 3.2$] \mu \mathrm{g} / \mathrm{mL}$ and 6.6 [IQR: 5.5$] \mu \mathrm{g} / \mathrm{mL}$, respectively; $p<0.001$ ). Female patients with an $\mathrm{HDL}-\mathrm{C} \geq 50 \mathrm{mg} / \mathrm{dL}$ had higher adiponectin levels than female patients with an HDL-C $<50 \mathrm{mg} / \mathrm{dL}$ (7.8 [IQR: 6.5$] \mu \mathrm{g} / \mathrm{mL}$ and 5.9 [IQR: 5.4$] \mu \mathrm{g} / \mathrm{mL}$, respectively; $\mathrm{p}<0.038$ ).

To further evaluate the characteristics of patients with discordance and concordance of LDL-C and non-HDL-C, the patients were classified into 4 subgroups: Group 1: LDL-C $<$ the median and non-HDL-C $<$ the median, group $2: \mathrm{LDL}-\mathrm{C}<$ the median and non-HDL-C $\geq$ the median, group 3: LDL-C $\geq$ the median and non-HDL-C $<$ the median, and group 4: LDL-C $\geq$ the median

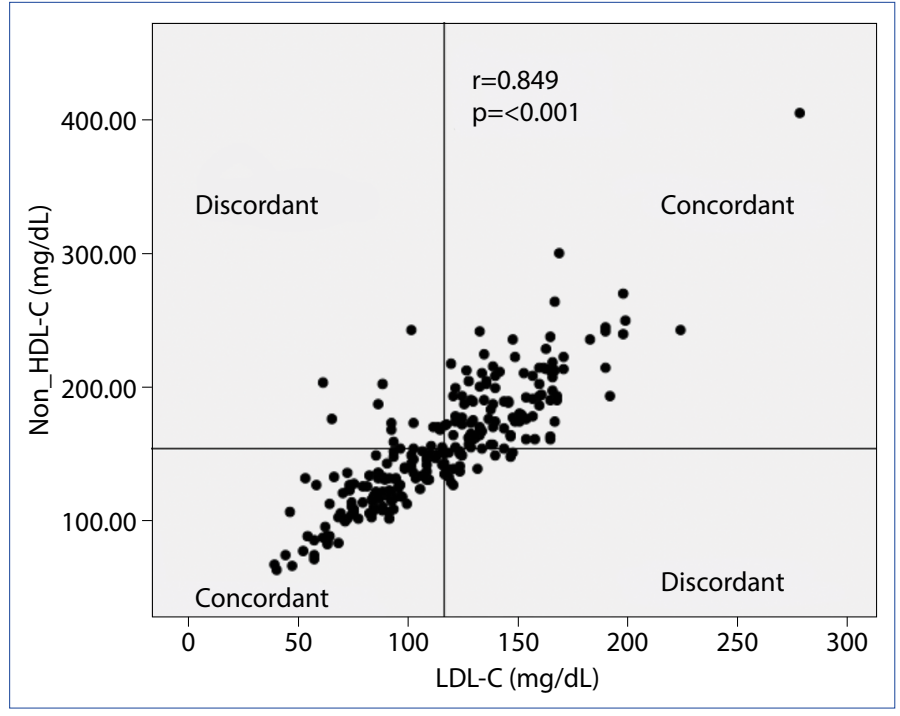

Figure 1. Scatterplot and prevalence of discordance and concordance defined according to median values of LDL-C and non-HDL-C.

HDL-C: High-density lipoprotein cholesterol; LDL-C: Low-density lipoprotein cholesterol.

and non-HDL-C $\geq$ the median. Groups 2 and 3 were discordant (Table 2). The variables of age, gender, BMl, smoking history, and the percentage of patients with hypertension were not significantly different between the groups. The patients in group 2 (LDL-C $<$ the median and non-HDL-C $\geq$ the median) had the highest prevalence of diabetes mellitus ( $p=0.007$ ). Although the median adiponectin measurement was lowest in group 2, the TG levels were highest, and the median ApoE was lowest in group 3 (LDL-C $\geq$ the median and non-HDL-C $<$ the median), there were no statistically significant differences between groups in the ApoE or adiponectin levels $(p=0.186$ and $p=0.161$, respectively).

\section{Discussion}

In the present study, we assessed the cross-sectional association between LDL-C and non-HDL-C discordance and the serum adiponectin and $A p o E$ concentrations in patients with significant CAD. The serum levels of adiponectin and ApoE in the discordant and concordant groups were not significantly different.

A sizeable proportion of patients have discordant LDL-C and non-HDL-C. The discordance rate in our study was $14 \%$, which is similar to that of previous studies [13-15]. It has been suggested that the LDL-C alone may give a false sense of security in one-fifth of diabetic subjects with a low LDL-C level, and patients with a normal LDL-C level may still be at risk for cardiovascular events [20]. Non-HDL-C is the second target for lipidlowering therapy and represents the cholesterol content of all circulating atherogenic lipoproteins [21]. It has been demonstrated that discordance of LDL-C and non-HDL-C either underestimates or overestimates coronary risk [14]. In particular, studies that follow up with patients who have a low LDL-C and 
Table 2. Characteristics of patients with concordant and discordant LDL-C and non-HDL-C levels

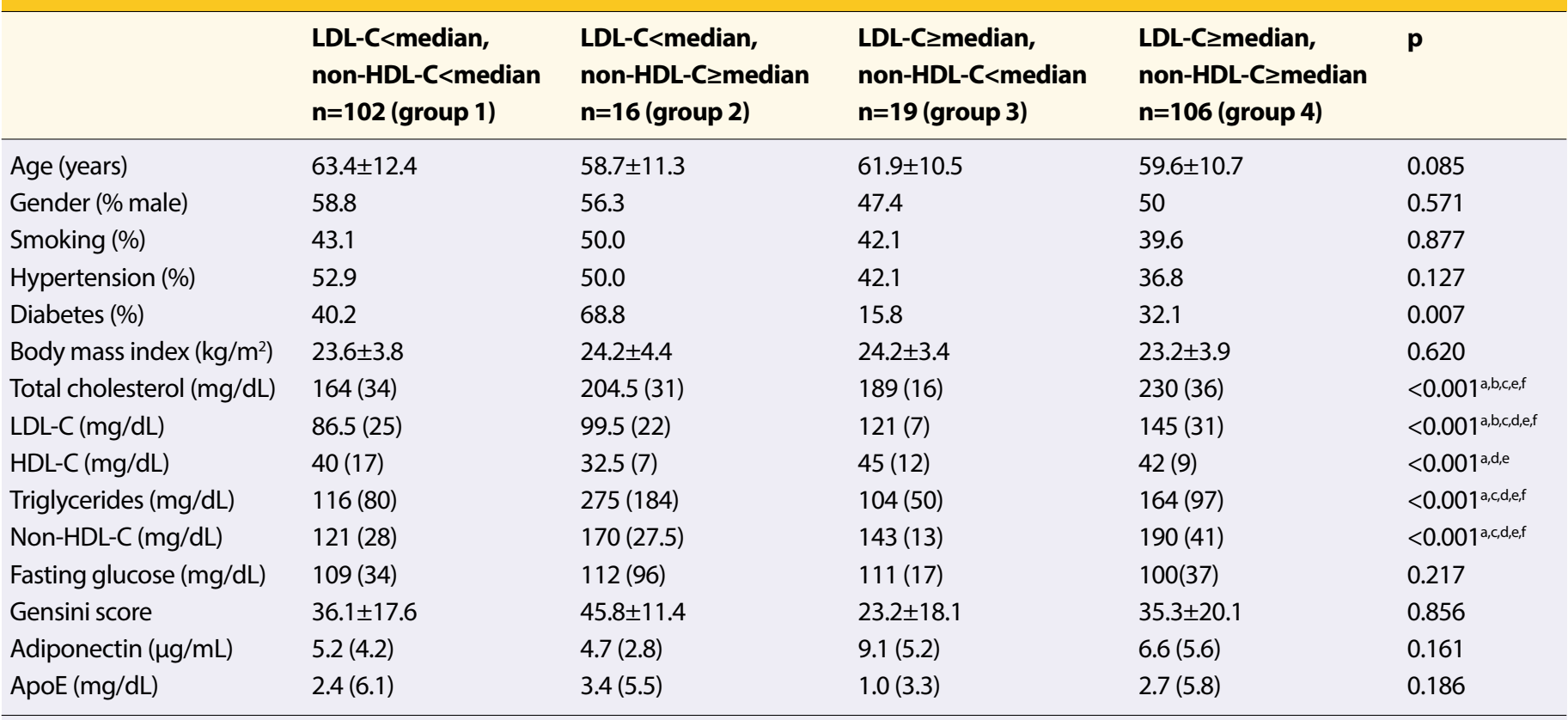

Data are expressed as percentage for categorical variables; chi-squared test was used. Data are presented as mean \pm SD or median (interquartile range) for continuous variables, and one-way analysis of variance or Kruskal-Wallis test were used to compare continuous measures. Statistically significant p values are in bold. The Bonferroni or Conover-Inman test was performed for binary comparisons between groups, and the $\mathrm{p}$ value was set at 0.05 . Significant differences were found between a) group 1 vs group 2 , b) group 1 vs group 3, c) group 1 vs group 4, d) group 2 vs group 3, e) group 2 vs group 4, and f) group 3 vs group 4. ApoE: Apolipoprotein E; HDL-C: High-density lipoprotein cholesterol; LDL-C: Low-density lipoprotein cholesterol.

a high non-HDL-C or vice versa and evaluate the impact of discordance on a CAD prognosis are currently lacking.

Discordance of LDL-C and non-HDL-C has been associated with obesity, glucose abnormalities, hypertriglyceridemia, and low HDL levels in previous studies [14, 15]. Adiponectin and ApoE levels have also been found to be associated with similar risk factors. A low plasma concentration of adiponectin has been observed in patients with metabolic syndrome, obesity, and diabetes mellitus $[10,11]$. ApoE levels increased in parallel with the number of metabolic syndrome components [6]. Both ApoE and adiponectin were associated with CAD and serum lipids. To further investigate the differences in CAD patients with discordant and concordant LDL-C and non-HDL-C, we evaluated plasma the ApoE and adiponectin concentrations.

Among lipoprotein abnormalities, a high LDL-C is major predictor of atherosclerosis, but many other hidden lipoprotein alterations may have a potential role in the pathogenesis of atherosclerosis. In a study by Barbagallo et al. [7], it was reported that patients with CAD had a higher concentration of ApoE-enriched TRLs than controls, despite both groups having similar TG, LDL-C, and non-HDL-C levels. The results of another study revealed that $C A D$ patients had a lower concentration of ApoE-containing HDL than the subjects without CAD, though both had similar total cholesterol and TG levels [22].

Plasma lipids affect the concentration of plasma ApoE. In normolipidemic subjects, the majority of serum ApoE resides in the HDL, whereas in hypertriglyceridemic subjects, it re- sides in the TRLs [4]. Total ApoE, TRL-ApoE and intermediatesized remnant-like lipoprotein-ApoE values were found to be higher in patients with hyperlipidemia than normolipidemic subjects [4]. The serum total ApoE concentration was significantly higher in low HDL-C subjects in a study that assessed ApoE at the extreme ends of HDL-C distribution [6]. We evaluated the serum ApoE concentration in patients with CAD and we further divided the patients into subgroups of those with discordant or concordant LDL-C and non-HDL-C. CAD severity was measured with the Gensini score and it was observed that the serum ApoE levels and the Gensini score did not differ significantly between the discordant and concordant groups. The ApoE value was numerically lowest (not statistically significant) in the group with the highest HDL-C and the lowest TG, but it was numerically highest in the group with the highest non-HDL-C and the highest LDL-C, rather than the group with the lowest HDL-C and the highest TG measurement.

Experimental studies have shown that adiponectin demonstrated antiatherosclerotic properties [23]. Also, a low serum adiponectin level has been associated with an increased risk of CAD in initially healthy subjects $[24,25]$. In contrast to studies including healthy subjects, a high serum adiponectin level has been associated with increased mortality in patients with manifest CAD in many studies, including a meta-analysis [2628]. The underlying mechanism of the adiponectin paradox in experimental studies of patients with and without CAD is still to be fully understood. Adiponectin has also been linked to 
dyslipidemia. A high plasma concentration of adiponectin has been associated with increased HDL-C, increased apolipoprotein $A 1$, and total cholesterol $[12,27,29]$. Moreover, it has been inversely correlated with serum TG and apolipoprotein B [12, $27,29]$. While the relationship between adiponectin and LDL-C is unclear, adiponectin has been found to be associated with smaller LDL particle size [30, 31]. In the present study, consistent with earlier studies, patients with a low HDL-C or high TG level had lower adiponectin levels than patients with a high HDL-C or a low TG level. The median LDL-C and non-HDL-C cutoffs used in our study indicated that the adiponectin was no longer significantly different between the discordant and concordant groups. It was numerically lowest (not statistically significant) in the discordant group with the lowest HDL-C and the highest TG.

\section{Study limitations}

This study has several limitations, such as the lack of measurement of ApoE subfractions or various isoforms of adiponectin. In addition, the plasma TRLs may or may not have contained ApoE, and the TRL or HDL may have been ApoE-poor or ApoErich. ApoE subfractions may differ in discordant and concordant groups. There is no absolute definition or standard cutoff value for the discordance of LDL-C and non-HDL-C. We used the median values of our study population. Other limitations include the small sample size and multiple factors that can affect plasma adiponectin levels and lipoprotein metabolism, including gender, age, dietary factors, and lifestyle. Age and gender did not differ between the discordant and concordant groups, but we did not assess dietary factors or lifestyle parameters. Considering the unmeasured potentially confounding factors in the population studied, the findings of our study should be interpreted as suggestive and additional, large-scale studies would be required to validate our results.

\section{Conclusion}

Among patients with significant CAD, $14 \%$ demonstrated a discordance of LDL-C and non-HDL-C. While high risk features, such as diabetes mellitus, hypertriglyceridemia, a higher total cholesterol and a higher LDL-C level differed between the discordant and concordant groups, the ApoE and adiponectin values were not significantly different. Adiponectin was negatively correlated with TG and positively correlated with $\mathrm{HDL}-\mathrm{C}$.

Conflict of Interest: The authors have no conflict of interest to declare.

Ethics Committee Approval: The research protocol was approved by Istanbul University Faculty of Medicine Clinical Research Ethics Committee on October 13, 2017 (No: 16).

Financial Disclosure: This research did not receive any other supplemental funding.
Peer-review: Externally peer-reviewed.

Authorship Contributions: Concept - O.K.; Design - O.K., A.F.E.; Supervision - N.C., A.K., E.A.; Funding - None; Materials - O.K., A.F.E., N.C.; Data collection \&/or processing - O.K., N.C., A.K., E.A.; Analysis and/or interpretation - O.K., A.F.E.; Literature search O.K.; Writing - O.K.; Critical review - N.C., A.K., E.A.

\section{References}

1. Kastelein JJ, van der Steeg WA, Holme I, Gaffney M, Cater NB, Barter P, et al; TNT Study Group; IDEAL Study Group. Lipids, apolipoproteins, and their ratios in relation to cardiovascular events with statin treatment. Circulation 2008;117(23):30029. [CrossRef]

2. Marais AD. Apolipoprotein E in lipoprotein metabolism, health and cardiovascular disease. Pathology 2019;51(2):165-76.

3. Gibson JC, Rubinstein A, Bukberg PR, Brown WV. Apolipoprotein $E$-enriched lipoprotein subclasses in normolipidemic subjects. J Lipid Res 1983;24:886-98. [CrossRef]

4. Cohn JS, Tremblay M, Amiot M, Bouthillier D, Roy M, Genest $\mathrm{J} \mathrm{Jr}$, et al. Plasma concentration of apolipoprotein $\mathrm{E}$ in intermediate-sized remnant-like lipoproteins in normolipidemic and hyperlipidemic subjects. Arterioscler Thromb Vasc Biol 1996;16(1):149-59. [CrossRef]

5. Yu H, Zhang W, Yancey PG, Koury MJ, Zhang Y, Fazio S, et al. Macrophage apolipoprotein $\mathrm{E}$ reduces atherosclerosis and prevents premature death in apolipoprotein $\mathrm{E}$ and scavenger receptor-class BI double-knockout mice. Arterioscler Thromb Vasc Biol 2006;26(1):150-6. [CrossRef]

6. Söderlund S, Watanabe H, Ehnholm C, Jauhiainen M, Taskinen MR. Increased apolipoprotein E level and reduced high-density lipoprotein mean particle size associate with low highdensity lipoprotein cholesterol and features of metabolic syndrome. Metabolism 2010;59(10):1502-9. [CrossRef]

7. Barbagallo $C M$, Rizzo $M$, Noto $D$, Frasheri $A$, Pernice V, Rubino $A$, et al. Accumulation of apoE-enriched triglyceriderich lipoproteins in patients with coronary artery disease. Metabolism 2006;55:662-8. [CrossRef]

8. Ouchi N, Kihara S, Arita Y, Nishida M, Matsuyama A, Okamoto $\mathrm{Y}$, et al. Adipocyte-derived plasma protein, adiponectin, suppresses lipid accumulation and class A scavenger receptor expression in human monocyte-derived macrophages. Circulation 2001;103(8):1057-63. [CrossRef]

9. Ouchi N, Kihara S, Arita Y, Maeda K, Kuriyama H, Okamoto Y, et al. Novel modulator for endothelial adhesion molecules: adipocyte-derived plasma protein adiponectin. Circulation 1999;100(25):2473-6. [CrossRef]

10. Arita Y, Kihara S, Ouchi N, Takahashi M, Maeda K, Miyagawa J, et al. Paradoxical decrease of an adipose-specific protein, adiponectin, in obesity. Biochem Biophys Res Commun 1999;257(1):79-83. [CrossRef]

11. Hotta K, Funahashi T, Arita $Y$, Takahashi M, Matsuda $M$, Okamoto $Y$, et al. Plasma concentrations of a novel, adiposespecific protein, adiponectin, in type 2 diabetic patients. Arterioscler Thromb Vasc Biol 2000;20(6):1595-9. [CrossRef] 
12. Oliveira GB, França Jí, Piegas LS. Serum adiponectin and cardiometabolic risk in patients with acute coronary syndromes. Arq Bras Cardiol 2013;101(5):399-409. [CrossRef]

13. Elshazly MB, Martin SS, Blaha MJ, Joshi PH, Toth PP, McEvoy JW, et al. Non-high-density lipoprotein cholesterol, guideline targets, and population percentiles for secondary prevention in 1.3 million adults: the VLDL-2 study (very large database of lipids). J Am Coll Cardiol 2013;62(21):1960-5. [CrossRef]

14. Mora S, Buring JE, Ridker PM. Discordance of low-density lipoprotein (LDL) cholesterol with alternative LDL-related measures and future coronary events. Circulation 2014;129(5):553-61. [CrossRef]

15. Kurmus O, Erkan AF, Ekici B, Aslan T, Eren M. Discordance of low-density lipoprotein cholestrol and non-high-density lipoprotein cholestrol and coronary artery disease severity. Arq Bras Cardiol 2020;114(3):469-75.

16. Gensini GG. A more meaningful scoring system for determining the severity of coronary heart disease. Am J Cardiol 1983;51(3):606. [CrossRef]

17. Chruściel $P$, Sahebkar $A$, Rembek-Wieliczko $M$, Serban $M C$, Ursoniu S, Mikhailidis DP, et al; Lipid and Blood Pressure Meta-analysis Collaboration (LBPMC) Group. Impact of statin therapy on plasma adiponectin concentrations: A systematic review and meta-analysis of 43 randomized controlled trial arms. Atherosclerosis 2016;253:194-208. [CrossRef]

18. Alaupovic P, Hodis HN, Knight-Gibson C, Mack WJ, LaBree L, Cashin-Hemphill $L$, et al. Effects of lovastatin on ApoA- and ApoB-containing lipoproteins. Families in a subpopulation of patients participating in the Monitored Atherosclerosis Regression Study (MARS). Arterioscler Thromb 1994;14:190613. [CrossRef]

19. Maeda N, Takahashi M, Funahashi T, Kihara S, Nishizawa H, Kishida $\mathrm{K}$, et al. PPARgamma ligands increase expression and plasma concentrations of adiponectin, an adipose-derived protein. Diabetes 2001;50(9):2094-9. [CrossRef]

20. Modi KD, Chandwani R, Ahmed I, Kumar KV. Discordance between lipid markers used for predicting cardiovascular risk in patients with type 2 diabetes. Diabetes Metab Syndr 2016;10(1 Suppl 1):S99-S102. [CrossRef]

21. Mach F, Baigent C, Catapano AL, Koskinas KC, Casula M, Badimon L, et al; ESC Scientific Document Group. 2019 ESC/
EAS Guidelines for the management of dyslipidaemias: lipid modification to reduce cardiovascular risk. Eur Heart J 2020;41(1):111-88. [CrossRef]

22. Wilson HM, Patel JC, Russell D, Skinner ER. Alterations in the concentration of an apolipoprotein E-containing subfraction of plasma high density lipoprotein in coronary heart disease. Clin Chim Acta 1993;220:175-87. [CrossRef]

23. Brochu-Gaudreau K, Rehfeldt C, Blouin R, Bordignon V, Murphy BD, Palin MF. Adiponectin action from head to toe. Endocrine 2010;37(1):11-32. [CrossRef]

24. Pischon T, Girman CJ, Hotamisligil GS, Rifai N, Hu FB, Rimm EB. Plasma adiponectin levels and risk of myocardial infarction in men. JAMA 2004;291(14):1730-7. [CrossRef]

25. Kumada M, Kihara S, Sumitsuji S, Kawamoto T, Matsumoto S, Ouchi N, et al; Osaka CAD Study Group. Coronary artery disease. Association of hypoadiponectinemia with coronary artery disease in men. Arterioscler Thromb Vasc Biol 2003;23(1):85-9. [CrossRef]

26. Schnabel R, Messow CM, Lubos E, Espinola-Klein C, Rupprecht $\mathrm{HJ}$, Bickel C, et al. Association of adiponectin with adverse outcome in coronary artery disease patients: results from the AtheroGene study. Eur Heart J 2008;29(5):649-57. [CrossRef]

27. Hascoet S, Elbaz M, Bongard V, Bouisset F, Verdier C, Vindis C, et al. Adiponectin and long-term mortality in coronary artery disease participants and controls. Arterioscler Thromb Vasc Biol 2013;33(1):e19-29. [CrossRef]

28. Wu ZJ, Cheng YJ, Gu WJ, Aung LH. Adiponectin is associated with increased mortality in patients with already established cardiovascular disease: a systematic review and meta-analysis. Metabolism 2014;63(9):1157-66. [CrossRef]

29. von Eynatten M, Hamann A, Twardella D, Nawroth PP, Brenner $\mathrm{H}$, Rothenbacher $\mathrm{D}$. Relationship of adiponectin with markers of systemic inflammation, atherogenic dyslipidemia, and heart failure in patients with coronary heart disease. Clin Chem 2006;52(5):853-9. [CrossRef]

30. Izadi V, Farabad E, Azadbakht L. Epidemiologic evidence on serum adiponectin level and lipid profile. Int J Prev Med 2013;4(2):133-40.

31. Weiss R, Otvos JD, Flyvbjerg A, Miserez AR, Frystyk J, Sinnreich $\mathrm{R}$, et al. Adiponectin and lipoprotein particle size. Diabetes Care 2009;32(7):1317-9. [CrossRef] 